\title{
Pre-Formulation and Formulation Approaches for Buccal Films
}

\author{
Chalamaiah $\mathbf{M}^{*}$ and Sharma PK \\ Food and Drug Toxicology Research Centre, National Institute of Nutrition (ICMR), Tarnaka, Hyderabad, India
}

\begin{abstract}
Many potential active pharmaceutical ingredients (APIs), which are drugs of abuse, or as recreational drugs, and which have narrow therapeutic index causing hundreds of deaths in recent years can be casted into buccal films. Many drugs which are lipophilic, and which can easily penetrate the blood-brain barrier can be of choice for buccal delivery. The bio-adhesive film for buccal delivery has advantage of the control delivery and has higher permeability, and lower reservoir capacity, of the epithelium. A simple system, consisting of a drug-loaded bio-adhesive coated onto an impermeable backing layer, can be easily envisaged.
\end{abstract}

Keywords: Active pharmaceutical ingredients; Blood-brain barrier; Medicines; Healthcare

\section{Introduction}

There are many potent, synthetic opioid analgesic drugs, which can be delivered as buccal films like fentanyl, a strong agonist of $\mu$-opioid receptors [1]. Many potential active pharmaceutical ingredients (APIs), which are drugs of abuse, or as recreational drugs, and which have narrow therapeutic index causing hundreds of deaths in recent years can be casted into buccal films [2-5]. It can be formulation of choice for drugs causing health problems due to over dose and improper medical use. It has been used for many potent analgesics, and it tends to induce nausea, as well as histamine-mediated itching. These films can also be used to produce prolonged drug release. In July 2014, the Medicines and Healthcare Products Regulatory Agency (MHRA) issued a warning regarding the potential for serious harm from accidental exposure to transdermal fentanyl patches, particularly in children, and directed that they should be folded, with the adhesive side in, before being thrown away, this unveils the importance of buccal film, which can be used as appropriate delivery for these type of drugs. These patches can be made tamper proof for children, who are most at risk from API overdose. Many deaths from drug overdose through buccal tablets and others have led a public health crisis recently, and many potent APIs continues to be a major killer drugs [5-7].

Many drugs which are lipophilic, and which can easily penetrate the blood-brain barrier can be of choice for buccal delivery. The drugs with very narrow effective plasma concentrations and short duration of action can be made into buccal films. Those drugs which have fast and high metabolism with more than $80 \%$ of an intravenous dose and getting metabolized within 30 min after administration can be administered via the buccal mucosa [8]. The transdermal approach when used for highly potent drug can result in its deposition in the skin and a significant delay (20-48 h) before effective plasma concentrations are reached (necessitating the use of a simultaneous treatment during this lag phase). Buccal delivery has potential to circumvent some of these problems and can be used for the immediate treatment. The bioadhesive film for buccal delivery has advantage of the control delivery and has higher permeability, and lower reservoir capacity, of the epithelium. A simple system, consisting of a drug-loaded bio-adhesive coated onto an impermeable backing layer, can be easily envisaged [9].

\section{Pre-Formulation Design}

For buccal films, the basic requirement for film is that it should have size of $1-3 \mathrm{~cm}^{2}$ and maximum duration of buccal delivery is $4-6 \mathrm{~h}$. a) Pharmaceutical considerations: It requires an excessive attention to develop safe and effective buccal adhesive film of fentanyl. The factors influencing the drug release, penetration through buccal mucosa, organoleptic factors and proper use of additives to improve the drug release and absorption along with effect of local irritation caused at the site of application should be considered while developing the buccal film formulation [10-15].

b) Buccal adhesive polymers: The buccal adhesive polymers have adhesive component in its property. They are generally hydrophilic and in dry form draw water from biological surface. This lead to strong interaction and form viscous liquid, which increases their retention time over mucosal surface, leading to adhesive phenomenon. They can be hydrogels like poly vinyl alcohol, sodium alginate, hydroxyl propyl methyl cellulose (HPMC), etc. They can be copolymers like graft copolymer of polystyrene and poly butadiene. They can also be multi-functional polymers like polyacrylates, chitosan etc. and others like carbopol/ carbomer, sodium carboxy methyl cellulose (CMC) [16-18]. These polymers have to be characterized with API or drug and the following interaction studies need to be performed:

\section{Drug-polymer interaction studies}

Using Fourier transform infrared spectroscopy (FTIR) and differential scanning calorimetry (DSC).

\section{Drug-polymer-excipient interaction studies}

Using FTIR and DSC.

\section{Drug release from polymer matrix}

Using dissolution testing apparatus and analytical techniques like high performance liquid chromatography (HPLC)/liquid chromatography-mass spectrometry (LC-MS).

*Corresponding author: Chalamaiah M, Food and Drug Toxicology Research Centre, National Institute of Nutrition (ICMR), Tarnaka, Hyderabad-500604 India, Tel: +914027197200; E-mail: akushwaha@go.olemiss.edu

Received August 18, 2016; Accepted September 02, 2016; Published September 10, 2016

Citation: Chalamaiah M, Sharma PK (2016) Pre-Formulation and Formulation Approaches for Buccal Films. J Bioequiv Availab 8: 246-248. doi: 10.4172/ jbb.1000303

Copyright: $(2016$ Chalamaiah M, et al. This is an open-access article distributed under the terms of the Creative Commons Attribution License, which permits unrestricted use, distribution, and reproduction in any medium, provided the original author and source are credited. 


\section{Stability studies}

Using simulated mucous fluid and stability chamber and HPLC or other analytical techniques like UV spectrophotometer, FTIR, etc.

Physiological considerations: The physiological consideration like texture of buccal mucosa, thickness of mucus membrane, its turn over time, and effect of salivary secretions play vital role in performance of API buccal film formulations. Effect of salivary enzymes on API stability and release should be considered before designing the API buccal film. It can be done by in-vitro mucous enzymatic degradation and stability studies.

Pharmacological consideration: The API absorption occurs through transcellular route as it is a lipophilic drug. Controlling and optimizing the $\mathrm{pH}$ can increase the drug interaction and can enhance the API permeability. Local residence time, concentration of drug in mucosa, and the amount of API transported into blood are responsible for its local or systemic delivery. Incorporation of permeation enhancers and enzyme inhibitors can improve the bioavailability of fentanyl. It can be done using in-vitro permeation studies and drug deposition studies.

Permeation enhancers: The selection of proper permeation enhancers can increase the efficacy of a drug. There are different permeation enhancer's available, so proper screening techniques and lead selection is important. It can be done using high throughput screening of permeation enhancers and analyzing the drug content using HPLC.

Drug loading and entrapment efficiency studies: It is done to quantify the amount of API present in specific size patch. HPLC can be used to quantify the fentanyl.

The XRD studies: XRD studies can be used to characterize the API buccal film.

After considering all these factors, the stability of lead excipients and API in the buccal environment and shelf life has to be accounted.

\section{Formulation Design}

The design of experiment can be used to prepare and optimize the formulation. Based on pre-formulation data, we can know the amount of drug and excipient can be used to prepare the API buccal film. We have different factorial designs like central composite design, $2^{2}, 2^{3}, 2^{4}$, etc. available in software which can be employed based on requirement, and can give us variability study data and can optimize and validate the formulation. The formulation characterization involves the following approaches:

\section{Preparation of API buccal film formulation and casting}

Conventional film casting method: The film casting method is the most widely used manufacturing process employed for making films. It's mainly due to the ease of the process and the low cost with the system setup incurs at the laboratory scale. The process consists of the following six basic steps: 1 . Preparation of the casting solution; 2 . Deareation of the solution; 3 . Transfer of the appropriate volume of solution into a mold; 4 . Drying the casting solution; 5. Cutting the final dosage form to contain the desired amount of drug; and 6. Packaging. During the manufacturing process of films, particular importance is given to the rheological properties of the solution or suspension, air bubbles entrapped, content uniformity, and residual solvents in the final dosage form. The rheology of the solution to be casted will determine the drying rates and uniformity in terms of the API as well as the physical appearance of the films. During the mixing steps of the manufacturing process, air bubbles are unintentionally introduced to the liquid and removal of air is a critical step for homogeneity of film. Films cast from aerated solutions exhibit an uneven surface and heterogeneous thickness [19-21].

Hot melt extrusion film: In hot-melt extrusion, a blend of API and other pharmaceutical ingredients are molten and then forced through an orifice (the die) to yield a more homogeneous material in different shapes, such as granules, tablets, or films. Hot-melt extrusion has been used for the manufacture of controlled-release matrix tablets, pellets, and granules, as well as orally disintegrating films. However, only a handful of articles have reported the use of hot-melt extrusion for manufacturing mucoadhesive buccal films. Repka and coworkers have extensively worked on the use of hot-melt extrusion for the manufacture of mucoadhesive buccal films, evaluating different matrix formers and additives for the processing of the blend $[15,16]$.

\section{Characterization of buccal film}

Drug loading efficiency: The specific size of film can be selected in triplicate and dissolved in appropriate solvent to completely solubilize the film. The API can be extracted and quantified using analytical techniques like HPLC, LC-MS, etc.

Film muco-adhesive property: Most of the muco-adhesive phenomena have two main stages that control the performance of the dosage form: the contact stage and the consolidation stage. The strength of the muco-adhesive bond will then be determined by the extent of intermixing that occurs after water migrates and reaches equilibrium. Texture analyzer is used to measure the muco-adhesion strength of buccal films that utilized chicken pouch as the biological membrane upon which the films were allowed to adhere. The instrument measures detachment forces from its mobile arm, after which normalizing is considered as adhesive forces, and the maximum force is normally referred to as muco-adhesive force. The use of this type of texture analyzer for the measurement of muco-adhesion on different dosage forms, such as buccal tablets, has already been reported. The previous research had focused on the importance of the method variables, which ultimately determine, together with the film and the substrate properties, the value of muco-adhesion strength $[9,13]$.

Tackiness and tensile strength of film: Besides the important parameter of muco-adhesion strength and residence time of buccal films, the mechanical properties play a crucial role on the physical integrity of the dosage form. Several values can be obtained from a regular stress-strain curve; however, most relevant to the study of buccal films are the tensile strength, the elongation at break, and the elastic modulus, also known as Young's modulus. The determination of the mechanical properties of a buccal film is usually based on the ASTM D882 method and measured using instruments such as a texture analyzer. The tensile strength of a film is defined as the resistance of the material to a force tending to tear it apart and normally identified as the maximum stress in the stress-strain curve.

Drug release profile: Flow through cell is an alternative method to that already described to study the release of API from buccal films. The dissolution medium can be phosphate buffer saline (PBS, $\mathrm{pH}$ 7.4) at $37^{\circ} \mathrm{C}$. Uniform mixing of the medium can be maintained by magnetic stirring at $300 \mathrm{rpm}$. To provide unidirectional release, each API buccal film can be sandwiched between donor compartment and receiver compartment on a flow through cell.

Drug permeation profile: The experimental procedure typically involves the use of a diffusion cell, which can be either vertical, such as a Franz diffusion cell, or horizontally oriented, such as the side-by-side 
Citation: Chalamaiah M, Sharma PK (2016) Pre-Formulation and Formulation Approaches for Buccal Films. J Bioequiv Availab 8: 246-248. doi: $10.4172 / \mathrm{jbb} .1000303$

or using diffusion cell. In these cells, a donor compartment is separated from a receptor compartment by a membrane acting as the mucosa model. Conditions such as temperature, composition of the receptor and donor media, $\mathrm{pH}$, cell dimensions, and hydrodynamic conditions are normally controlled in these experiments.

Stability studies: The shelf life of the API buccal film can be performed using stability chambers as per USFDA guidelines [10,12].

In-vitro metabolism studies: The development of in vitro buccal epithelial cell culture models for testing drug metabolism is in progress. To culture buccal epithelial cells, the cells must be harvested and cultured under specific conditions with an appropriate growth medium, temperature and humidity. The cell cultures have been reported to be grown from hamster cheek pouch. These cell cultures, however, do not differentiate to form a complete keratinized layer as observed in the normal hamster cheek pouches and they subsequently have a greater permeability for compounds with reference to tissue source itself. Therefore, these hamster cheek cultured cells narrowly mimic the human buccal mucosa cells and so this can be a suitable model in predicting the permeability through the human buccal mucosa [8].

\section{In-vivo studies}

- In-vivo residence time: In vitro residence time may not be perfect prediction of in vivo behavior of buccal films hence the determination of in vivo residence time is often considered to be prerequisite to ensure the performance of dosage forms $[11,12]$. In vivo residence time tests are performed usually in animal models or humans. Patel and Poddar have used rabbits for measuring in vivo residence time. The rabbits were anesthetized to carry out experiment with plain as well as medicated patches. The muco-adhesive patch was placed on the buccal mucosa between the cheek and gingiva in the region of the upper canine and pressed gently onto the mucosa for about $30 \mathrm{sec}$. The time required for complete erosion of the patch or the time at which failure of adhesion occurred between the patch and mucosa was noted as in vivo residence time [5].

- Buccal absorption test: The simplest measurement to assess drug permeability across the buccal mucosa is 'buccal absorption test', also known as the 'swirl and spit test'. In this study, a quantified volume of a drug solution is administered into the oral cavity of a subject, who will swirl it around for a specified period of time and then expels it. The subject then rinses his or her mouth with distilled water or buffer solution, and the expelled drug solution and rinse are combined and analyzed for drug content. The difference between the initial and final drug concentration in the solution is assumed to be the amount of drug taken up into the oral mucosa [1].

- Pharmacokinetic study: Buccal absorption and perfusion studies usually provide an understanding of the potential of a formulation to deliver a drug but these lack real in vivo systemic evaluation and other physiological factors can contribute to overall performance of the API buccal film formulation. Hence, it is considered necessary to perform an in vivo pharmacokinetic study. The animal models like rabbit, dog and pig have been used so far to perform such initial in vivo studies before going to human trials [7].

\section{Conclusion}

The above mentioned pre-formulation and formulation design for buccal film can improve the efficacy and performance via muco- adhesive delivery. It will characterize the film completely and it can correlate to its real time in vivo efficacy.

\section{Reference}

1. Peng PWH, Sandler AN (1999) A review of the use of fentanyl analgesia in the management of acute pain in adults. Anesthesiology 90: 576-599.

2. Kushwaha A, Murthy RN, Murthy SN, Elkeeb R, Hui X, et al. (2015) Emerging therapies for the treatment of ungual onychomycosis. Drug Dev Ind Pharm 41: 1575-1581.

3. Gourlay GK (2001) Treatment of cancer pain with transdermal fentanyl. Lancet Oncology 2: 165-172.

4. Kushwaha A, Jacob M, Shiva Kumar HN, Hiremath S, Aradhya S, et al. (2015) Trans-ungual delivery of itraconazole hydrochloride by iontophoresis. Drug Dev Ind Pharm 41: 1089-1094.

5. del Consueloa IB, Falsonb F, Guyd RH, Jacquese Y (2007) Ex vivo evaluation of bio adhesive films for buccal delivery of fentanyl. Journal of Controlled Release 122: 135-140.

6. Kushwaha A, Shivakumar HN, Murthy SN (2016) lontophoresis for drug delivery into the nail apparatus: exploring hyponychium as the site of delivery. Drug Dev Ind Pharm 42: 1678-1682.

7. U S Food and Drug Administration. Drugs@FDA. FDA Approved Drug Products.

8. Kushwaha A, Manda P, Murthy SN (2013) Intranasal delivery of fluorocytosine.

9. https://en.wikipedia.org/wiki/Fentanyl

10. Manda P, Kushwaha AS, Kundu S, Shivakumar HN, Jo SB, et al. (2016) Delivery of ziconotide to cerebrospinal fluid via intranasal pathway for the treatment of chronic pain. J Control Release 224: 69-76.

11. Dixit R, Puthli S (2009) Oral strip technology: overview and future potential Journal of Controlled Release 139: 94-107.

12. Kushwaha A, Shivakumar HN, Murthy SN (2013) Reverse iontophoresis for Trans-Ungual Drug Delivery. AAPS.

13. Barnhart S, Rathbone MJ, Hadgraft J, Roberts MS, Lane ME (2008) Modifiedrelease Drug Delivery Technology, Second Edition. Informa Healthcare pp: 209-216.

14. Kushwaha A, Shivakumar HN, Murthy SN (2013) Losartan loaded hydroge micro particles for delivery of drugs to Chronic Wounds. AAPS.

15. Repka M, Prodduturi S, Stodghill S (2003) Production and characterization of hot-melt extruded films containing clotrimazole. Drug Development and Industrial Pharmacy 29: 757-765.

16. Repka M, McGinity J (2001) Bioadhesive properties of hydroxypropylcellulose topical films produced by hot-melt extrusion. Journal of Controlled Release 70 : 341-351.

17. Morales JO, McConville JT (2011) Manufacture and characterization of mucoadhesive buccal films. European Journal of Pharmaceutics and Biopharmaceutics 77: 187-199.

18. Kushwaha A, Shivakumar HN, Murthy SN (2013) Bioadhesive film forming formulation for topical delivery of drugs. AAPS.

19. Patela VF, Liua F, Browna MB (2012) Modeling the oral cavity: In vitro and in vivo evaluations of buccal drug delivery systems. Journal of Controlled Release 161: 746-756.

20. Patel RS, Poddar SS (2009) Development and characterization of mucoadhesive buccal patches of salbutamol sulphate. Curr Drug Deliv 6: 140-144.

21. Kushwaha A, Shivakumar HN, Murthy SN (2013) Conductive topical nail lacquer for transungual iontophoresis. AAPS. 